\title{
Infermieristica nefrologica: competenze assistenziali generali e specifiche
}

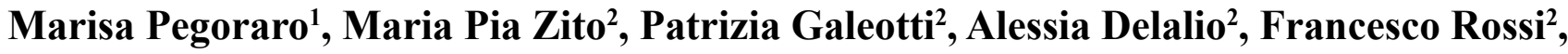 \\ Vincenza Guadagno ${ }^{3}$
}

${ }^{1}$ Presidente EDTNA/ERCA Filiale Italiana

${ }^{2}$ Segreteria Scientifica EDTNA/ERCA

${ }^{3}$ Coordinatore Infermieristico Nefrologia e Dialisi, Bologna

\begin{abstract}
NURSING COMPETENCES
Abstract. The article discusses the actual statement of the EDTNA/ERCA ITALIAN BRANCH related to Nephrology nursing advanced competences in the clinical setting, aiming at actively taking part to the national debate on the allocation of advanced nursing practice. Taking into account the ISFOL model as a definition of the work curricula proposed for the free circulation in both the EU and international areas, the model is elaborated to enhance healthcare core items and, more specifically, in the Nephrology nursing setting, starting from basic competences to healthcare, cross-sectional, and specific competences. The pathway moves from general to specific, defining 6 areas of Nephrology nursing interest (outpatients department, ward unit, peritoneal dialysis, hemodialysis, intensive care, and transplantation). For each area, nursing competences are listed (knowing, doing, being). The article is meant to be used as a guideline, benchmarking, and audit tool for the various national Nephrology nursing settings.
\end{abstract}

Key words: Nursing competences, Renal nursing

Conflict of interest: None.

Financial support: None.

Accettato: 17 Febbraio 2014

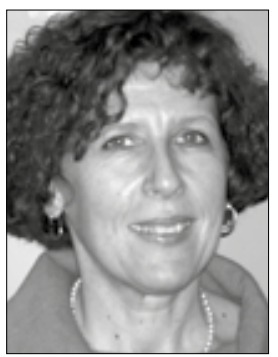

Marisa Pegoraro

\section{Introduzione}

La Filiale Italiana dell'EDTNA/ERCA, in quanto Associazione Nazionale di Area Nefrologica, vuole partecipare attivamente al dibattito sulla definizione delle competenze avanzate nell'infermieristica nazionale, definendo le specificità proprie dell'assistenza infermieristica ai pazienti nefropatici nell'ambito dei vari contesti/ambiti di cura. Le competenze qui espresse sono da intendere in termini di conoscenze, capacità/abilità e comportamenti come valore aggiunto del professionista, che vengono messi in atto in un determinato contesto per raggiungere uno scopo di benessere e cura, concordato anche con la persona assistita e il suo gruppo familiare.

Con la sovranità che caratterizza la scelta degli obiettivi in ogni Associazione, lo scambio culturale con i colleghi europei in corso da molti anni e la volontà di essere parte del panorama infermieristico nazionale, il Comitato Direttivo della Filiale ha elaborato una rappresentazione delle competenze agite e/o agibili nell'ambito dell'area nefrologica. Condividendo la posizione della Federazione Nazionale dei Collegi IPASVI e volendo concretamente supportare l'azione di avanzamento e promozione della professione infermieristica, vengono esposte qui di seguito le competenze che sono attività quotidiana nella maggioranza dei servizi nefrologici italiani. Tale rappresentazione è nata dall'esperienza sul campo, dai confronti avuti nei congressi nazionali, dalle argomentazioni scelte per le giornate di studio e da alcuni testi di riferimento generale. Ogni servizio potrà fare confronti tra quanto qui espresso e la propria realtà operativa. Siamo certi che, per tutti i centri, ci saranno aree in cui l'eccellenza è stata raggiunta e altre in cui il miglioramento è perseguibile.

\section{Materiali e metodi}

Per l'analisi delle competenze agite è stato utilizzato l'approccio ISFOL già adottato dalla Federazione IPASVI (1), molto prima che la discussione fosse spostata a livello di Commissione Stato-Regioni. Inoltre, dal momento che siamo Filiale di un'associazione europea e che esiste la libera circolazione della professione nei Paesi dell'Unione, è per noi prioritario 


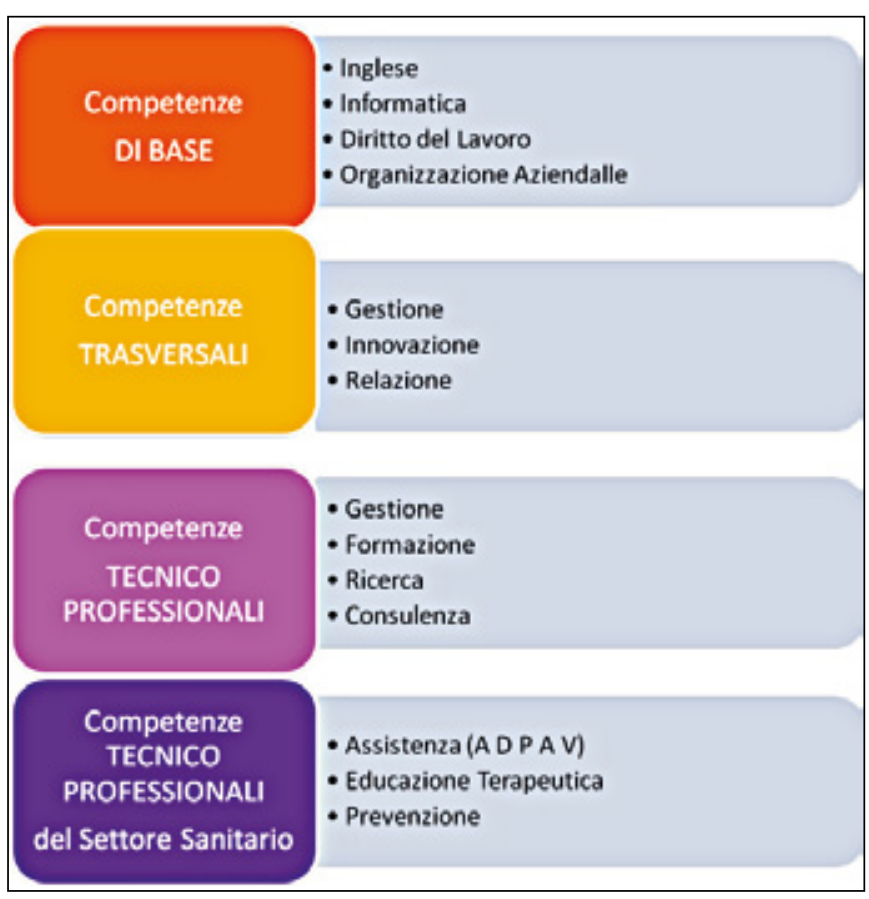

Fig. 1 - Le varie aree di competenza considerate dal modello ISPOL.

mantenere linguaggi uniformi e condivisibili, al di là della rappresentazione che gli organi nazionali della politica vorranno dare alla formalizzazione della professionalità espressa dall'infermieristica nazionale.

Il modello ISFOL (Istituto per lo Sviluppo della Formazione Professionale dei Lavoratori) è utilizzato in vari ambiti, e non solo in quello sanitario, e prevede la definizione di aree di attività specifiche per le singole professioni, di aree di base comuni e interprofessionali e, infine, di aree specifiche all'interno della stessa professione (Fig. 1).

Per la professione infermieristica, la Federazione aveva indicato 6 macro aree di competenza infermieristica, come riportate nella Tabella I.

\section{Il "nephrology nurse"}

L'associazione Europea EDTNA/ERCA ha definito il profilo del "NEPHROLOGY NURSE" già da qualche decennio, proponendo anche un Core Curriculum di Formazione Post Base a supporto delle competenze richieste nei vari ambiti clinicoassistenziali (2).

“... È un professionista della salute con adeguate conoscenze nell'assistenza a soggetti affetti da insufficienza renale ad ogni stadio della progressione terapeutica. È coinvolto nell'erogazione delle migliori cure disponibili per i pazienti e le loro famiglie. Provvede, in particolare, a supporto, educazione, prevenzione delle complicanze e riabilitazione, incoraggiando i pazienti verso l'indipendenza e l'autocura. L'EDTNA/ERCA mette a disposizione idee operative per raggiungere tali obiettivi. È membro di un gruppo multidisciplinare e, in questo, agisce come avvocato del paziente. È esperto clinico nell'assistenza nefrologica e contribuisce positivamente alla soddisfazione dei bisogni della comunità come professionista esperto del gruppo di cura il cui scopo è quello di assicurare al paziente il rispetto dei propri valori culturali, credo e dignità.

Interviene come consulente, ricercatore, agente di cambiamento e insegnante, integrando le proprie competenze di gestione, ricerca e formazione, per migliorare sia le condizioni di vita dei singoli individui affetti da insufficienza renale che $i$ servizi che ad essa provvedono".

(Baer, 1979) - EDTNA/ERCA Nephrology Nurse Profile 1994

\section{Elaborati prodotti}

\section{Aree generali}

L'assistenza nei vari ambiti clinici riconosce delle componenti della competenza professionale comuni ed imprescindibili dall'atto assistenziale, in qualsiasi punto del continuum di salute-malattia. In parte sono quelle tipiche della professione, già menzionate nella Tabella I, ma anche arricchite di alcuni elementi tipici dell'assistenza ai pazienti cronici e a quelle patologie che espongono anche i professionisti ai rischi della "cronicità" delle prassi e dei gesti assistenziali (Fig. 2).

\section{TABELLA I - AREE DI COMPETENZA TIPICHE DELLA PROFESSIONE INFERMIERISTICA}

\begin{tabular}{|c|c|}
\hline $\begin{array}{l}\text { ASSISTENZA } \\
\text { - Accertare } \\
\text { - Diagnosticare } \\
\text { - Pianificare } \\
\text { - Attuare } \\
\text { - Valutare }\end{array}$ & $\begin{array}{l}\text { GESTIONE } \\
\text { - Effettuare diagnosi organizzative } \\
\text { - Promuovere la cultura della qualità } \\
\text { - Pianificare il lavoro } \\
\text { - Gestire la privacy } \\
\text { - Gestire il rischio clinico } \\
\text { - Analizzare il contesto socio sanitario }\end{array}$ \\
\hline $\begin{array}{l}\text { EDUCAZIONE TERAPEUTICA } \\
\text { - Realizzare interventi di educazione terapeutica }\end{array}$ & $\begin{array}{l}\text { FORMAZIONE } \\
\text { - Progettare, erogare e gestire l'evento formativo } \\
\text { - Gestire l'ambito di apprendimento }\end{array}$ \\
\hline $\begin{array}{l}\text { PREVENZIONE } \\
\text { - Educare agli stili di vita } \\
\text { - Attuare programmi di prevenzione }\end{array}$ & $\begin{array}{l}\text { RICERCA } \\
\text { - Sviluppare un progetto di ricerca }\end{array}$ \\
\hline
\end{tabular}




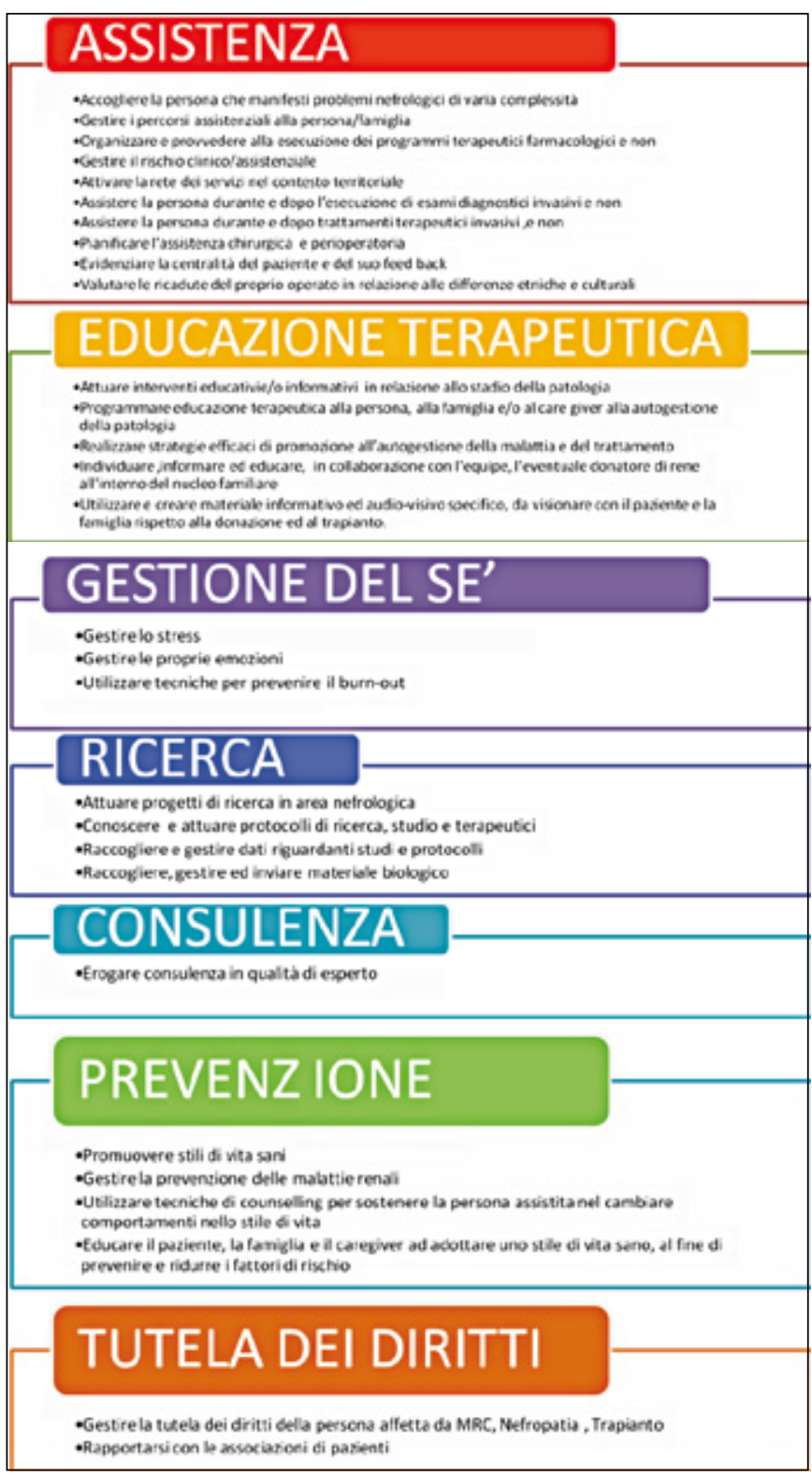

Fig. 2 - Componenti della competenza professionale.

\section{Aree specifiche}

Nell'ambito dell'infermieristica nefrologica, le competenze si espletano in diversi contesti di assistenza e cura (Fig. 3), in cui le componenti relazionali, tecniche e riabilitative hanno focus diversi, anche se simili.

È stata fatta un'analisi delle competenze per ogni area clinica, in cui sono esplicitati i contenuti della competenza specifica e le azioni che la rendono esplicita. In questo elaborato, non è stato affrontato l'argomento della mappatura qualitativa delle competenze, quella che Patricia Benner (3) indica da novizio a esperto, adottata, peraltro, anche dall'associazione europea, rimandando l'argomento a un prossimo elaborato.

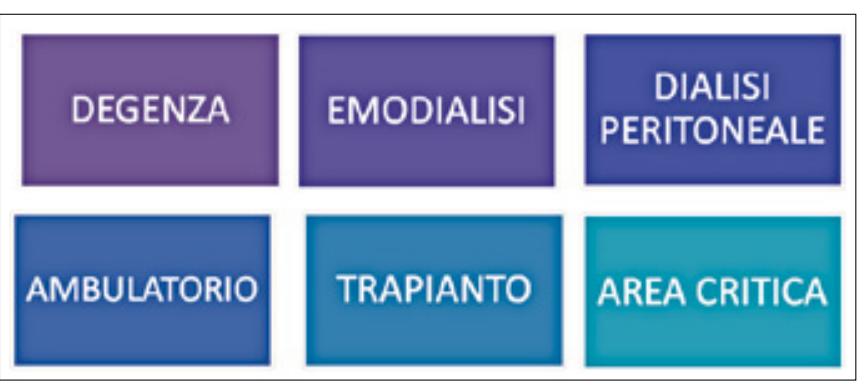

Fig. 3 - I contesti dell'assistenza infermieristica nefrologica.

\section{Analisi delle competenze specifiche}

\section{Area degenza}

Capacità di prendere in carico la persona che manifesti problemi nefrologici nei vari stadi ed espressioni della progressione della MRC

- Definire una diagnosi infermieristica clinica assistenziale

- Implementare i percorsi clinico assistenziali del nefropatico

- Pianificare interventi assistenziali al personale di supporto (OS, OSS, ecc.)

- Controllare i parametri vitali e riconoscere i segni di peggioramento della funzionalità renale

- Preservare il letto vascolare del paziente con malattia renale (valutazione per incannulare la vena, per i prelievi ecc.)

- Concorrere all'assunzione della dieta corretta

- Somministrare i farmaci nei tempi e nelle modalità specifici

Capacità di gestire il paziente portatore di sistemi di monitoraggio e/o somministrazione di trattamenti terapeutici o diagnostici

- Misurazione di alcuni parametri vitali in modalità invasiva (PVC); Somministrazione di farmaci in device multilume elo CVC da Dialisi sia temporaneo che permanente

- Gestione del drenaggio addominale(post intervento di TX renale)

- Gestione assistenziale di cateteri venosi periferici (PICC, Medline ecc.) e centrali e prevenzione delle infezioni correlate

- Gestione di FAV e cateteri peritoneali

- Assistenza al paziente in trattamento di aferesi terapeutica

- Conoscenza e attuazione di protocolli di cura specifici (biopsie, $A T G$ )

Capacità di gestire la relazione di aiuto con la persona assistita e la sua famiglia

- Relazionarsi con la persona assistita in nefrologia

- Educare i caregiver a gestire le cure nefrologiche

- Educare i caregiver a gestire la sopravvivenza della persona con malattia cronica

- Supportare le famiglie e la persona assistita nell'elaborazione del lutto

- Educare all'autocura la persona con problemi nefrologici

- Prevenire la malnutrizione nel paziente nefropatico 
Capacità di relazionarsi con gli altri operatori/servizi/ processi coinvolti nei vari percorsi diagnostico-terapeutici relativi

- Alla continuità assistenziale (dimissioni protette)

- Ai sistemi informativi sui processi assistenziali (cartelle informatizzate)

- Alle cure delle malattie rare (protocolli specifici)

- Alla gestione del dolore nella persona con malattia renale

- Al supporto nella prevenzione di eventi acuti e cronici (iperkaliemia, iperfosforemia, iperidratazione, aritmie) e di reazioni avverse ai farmaci

\section{Area ambulatorio (2 sezioni)}

a) Malattie genetiche E IMMUNOMEdiate CON INTEResSAMENTO NEFROLOGICO

Conoscere i percorsi diagnostico-terapeutici e i protocolli di ricerca, di studio e terapeutici in corso, conoscere e gestire i trial

- Saper effettuare diagnosi infermieristiche

- Raccogliere e gestire dati e materiale biologico

- Gestire terapie infusionali

- Conoscere la farmacocinetica della terapia in uso

- Applicare i protocolli di follow-up riconosciuti a livello internazionale

- Contattare direttamente i laboratori sul territorio nazionale per la spedizione dei materiali biologici e il ritiro del referto

\section{b) Ambulatorio Malattia Renale Cronica III/V stadio}

Conoscere i cardini dei trattamenti conservativi e sostitutivi per informare il paziente sull'eventuale scelta terapeutica nell'ambito di un percorso strutturato e proceduralizzato, sentendosi parte attiva dell'equipe multidisciplinare, stimolando aggiornamento e formazione

- Informare sugli effetti positivi e collaterali dei farmaci di uso più comune (EPO, chelanti del P, ipotensivi)

- Gestire il paziente in early e late referral

- Conoscere le tecniche di comunicazione

- Educare all'autocura la persona con problemi nefrologici

- Conoscere i percorsi di individuazione e formazione del caregiver

- Perseguire $i$ principi di pratica e teoria, $i$ suggerimenti e $i$ modelli alimentari e dietetico nutrizionali validi nel trattamento dietetico della MRC

- Controllare i referti ematochimici e strumentali e allertare il medico su eventuali azioni da compiere per ulteriori approfondimenti

Capacità di valutare la persona assistita e l'ambito familiare, al fine di cogliere possibilità di intervento mirato relativo allo stadio della MRC e alle scelte terapeutiche possibili

- Capacità di gestire la relazione di aiuto con la persona assistita e la famiglia

- Conoscenza dei percorsi diagnostico-terapeutici dei pazienti in early e late referral, dei protocolli di ricerca in corso, di studio e terapeutici

- Conoscenza dei cardini dei trattamenti conservativi e sostitutivi per informare il paziente sull'eventuale scelta terapeutica nell'ambito di un percorso strutturato e proceduralizzato

- Capacità di relazionarsi con gli altri operatori/servizi/processi coinvolti nei vari percorsi diagnostico-terapeutici relativi alla continuità assistenziale (presa in cura sul territorio)

Collaborare con le U.O.C. aziendali e creare corsie privilegiate o creare Percorsi Ambulatoriali Complessi, che favoriscano i processi diagnostici, curativi e assistenziali del paziente cronico e della donazione d'organi

- Gestire le cartelle cartacea e informatizzata, se esistono, e l'archivio

- Saper gestire la privacy

- Creare e divulgare del materiale informativo dedicato al paziente e alla sua famiglia

\section{Area trapianto}

Conoscere il percorso di trapiantologia e le Linee Guida del CNT (Centro Nazionale Trapianti) e i protocolli dei Centri Trapianto di riferimento, relative alla messa in lista per trapianto da cadavere e da vivente

- Conoscere e attuare le Linee Guida riguardanti il trapianto da vivente e da cadavere

- Individuare, informare ed educare l'eventuale donatore

- Utilizzare i percorsi ambulatoriali complessi o le corsie preferenziali per lo studio e per l'inserimento in lista TX

- Gestire gli esami ematochimici e strumentali e le visite di controllo nefrologiche presso il centro trapianti

- Assemblare e inviare, nei tempi richiesti, ai centri trapianto la scheda per l'inserimento in lista e le fotocopie di tutte le indagini effettuate dal paziente

- Coordinare la raccolta e l'invio dei sieri per il mantenimento in lista ogni tre mesi

Conoscere le tecniche di comunicazione ed educazione terapeutica e di gestione delle emozioni relative al trapianto da vivente e da cadavere

- Monitoraggio delle emozioni/sensazioni del donatore e successivo supporto

- Saper individuare eventuali momenti di panico, dubbio e sconforto nel donatore

- Capire se la donazione è spontanea o se è dovuta a eventuali forzature e comportarsi secondo le procedure

- Prevedere incontri di supervisione e formazione

- Creare e utilizzare del materiale informativo e visivo specifico, da visionare con il paziente e la famiglia

- Informare e supportare il paziente che non può effettuare il trapianto e invio all'equipe di riferimento

Capacità di accogliere il paziente nel post trapianto e seguire le direttive del centro trapianto di riferimento - Monitorare l'aderenza terapeutica del paziente ai trattamenti e allo stile di vita 
- Controllare i referti ematochimici e strumentali e allertare il medico su eventuali azioni da compiere per ulteriori approfondimenti

- Prevedere incontri di supervisione e formazione

- Supportare il paziente nella fase di esaurimento dell'organo trapiantato e di transizione alla dialisi

- Sentirsi sempre parte di un'equipe in ogni evenienza descritta in precedenza

\section{Area dialisi peritoneale}

Gestione del catetere peritoneale

- Conoscere i diversi tipi di catetere peritoneale

- Gestire la fase di preparazione all'inserzione del catetere peritoneale

- Conoscere le diverse tecniche di inserzione del catetere peritoneale e, se richiesto o necessario, assistere durante l'intervento di inserzione

- Gestire la fase post inserzione del catetere peritoneale

- Conoscere la procedura di medicazione dell'exit site in sintonia con le Linee Guida "ISPD Guideline"

- Prevenire le principiali complicanze post operatorie

- Riconoscere le principali complicanze post operatorie

- Gestire in autonomia e in collaborazione con il medico le principali complicanze post operatorie

- Effettuare correttamente la valutazione e la medicazione dell'exit site e segnalare tempestivamente eventuali alterazioni

- Riconoscere e gestire le complicanze infettive e non infettive relative al catetere peritoneale (leakage)

- Eseguire l'addestramento del paziente ed educarlo alla gestione del catetere peritoneale

- Verificare l'apprendimento delle conoscenze e della corretta tecnica di gestione del catetere peritoneale

Capacità di applicare le tecniche di dialisi peritoneale manuale e automatizzata

- Conoscere le diverse soluzioni dialitiche

- Conoscere i diversi set di connessione

- Conoscere la tecnica di connessione dei diversi set

- Conoscere le varie tipologie di tecniche dialitiche

- Conoscere i diversi tipi di cycler

- Impostare lo schema dialitico sui diversi cycler

- Valutare la persona assistita prima di effettuare una seduta dialitica

- Rilevare e segnalare la presenza di alterazioni in base alla situazione del paziente, prima di effettuare la seduta dialitica

- Applicare le prescrizioni del medico

- Monitorare la persona assistita durante la seduta di dialisi peritoneale

- Fornire tutoraggio telefonico al paziente per la risoluzione delle problematiche che non richiedono una valutazione ospedaliera

- Gestire le complicanze che richiedono una valutazione ospedaliera

- Risolvere i principali allarmi relativi alle diverse apparecchiature
Conoscenza delle complicanze legate alla dialisi peritoneale

- Riconoscere l'infezione peritoneale attraverso l'osservazione delle caratteristiche del liquido drenato

- Riconoscere le complicanze non infettive che alterano le caratteristiche dell'effluente peritoneale (chiloperitoneo, emoperitoneo)

- Riconoscere il malfunzionamento del catetere peritoneale

- Partecipare, per quanto di competenza, all'identificazione delle cause di malfunzionamento

Capacità di pianificare, attuare e valutare interventi di educazione terapeutica nel percorso di training della persona assistita

- Educare la persona assistita e il caregiver all'apprendimento della tecnica di connessione alla seduta dialitica, manuale o automatizzata

- Educare la persona assistita e il caregiver al riconoscimento di segni e sintomi di iperidratazione/disidratazione

- Educare la persona assistita e il partner a riconoscere le principali complicanze legate alla metodica dialitica

- Educare la persona assistita elo il partner alla gestione autonoma del trattamento dialitico domiciliare, manuale elo automatizzato in condizioni di completa autonomia e sicurezza

- Educare la persona assistita al controllo di peso corporeo, idratazione e dieta

- Pianificare e condurre interventi educativi mirati in relazione a specifici bisogni

- Verificare l'efficacia dell'intervento educativo, pianificando controlli rispetto al consolidamento delle competenze acquisite (re-training)

- Controllare i referti ematochimici e strumentali e, se necessario, allertare il medico per ulteriori approfondimenti

Capacità di pianificare e attuare interventi organizzativi miranti a soddisfare $i$ bisogni del malato cronico in trattamento dialitico domiciliare

- Organizzare le richieste e la consegna del materiale del trattamento al domicilio del paziente

- Coordinare l'eventuale messa in lista trapianto in collaborazione con l'ambulatorio/centro trapianti e i percorsi diagnostico-terapeutici correlati

- Riconoscere le problematiche individuali della persona assistita e le possibili cause di non compliance al programma dialitico previsto

- Organizzare e attuare visite a domicilio periodiche

- Organizzare momenti di re-training periodici o su necessità

\section{Area emodialisi}

Conoscenza tecnica delle apparecchiature dialitiche e loro funzionamento

\section{Conoscenza dell'impianto per la depurazione delle acque} di dialisi

- Applicare la procedura di monitoraggio, disinfezione e controllo dell'impianto di osmosi inversa secondo il protocollo concordato con le competenze tecniche di riferimento 
Capacità di valutare, durante la seduta, i parametri vitali dell'assistito e di monitorarne l'andamento, al fine di cogliere le possibili variazioni

- Impostare in autonomia il profilo dialitico come da prescrizione medica

- Condurre le sedute dialitiche nelle diverse modalità terapeutiche

- Effettuare la verifica visiva e il monitoraggio della persona assistita durante la seduta dialitica

- Risolvere prontamente i principali allarmi relativi alle diverse apparecchiature

- Eseguire correttamente la disinfezione dei monitor

Gestione accurata degli accessi vascolari

- Effettuare correttamente la "valutazione iniziale" della persona assistita

- Applicare correttamente i protocolli operativi relativi alla gestione degli accessi vascolari (FAV, protesi, CVC)

- Effettuare correttamente e secondo protocollo la medicazione del catetere venoso centrale

- Praticare la puntura della FAV applicando il protocollo di puntura scelto per il singolo paziente (rotazione dei siti, area, sito costante)

- Osservare e segnalare tempestivamente eventuali alterazioni dell'accesso vascolare (infezioni, trombosi)

- Eseguire correttamente l'emostasi e la medicazione della fistola artero-venosa

- Eseguire pratiche di monitoraggio dell'accesso vascolare (ricircolo, QBstress, ecodoppler funzionale) secondo quanto concordato e disponibile

Capacità di pianificare e attuare interventi di educazione terapeutica miranti a soddisfare i bisogni del malato cronico in trattamento dialitico

- Educare la persona assistita alla cura e alla gestione del proprio accesso vascolare in relazione alle potenzialità soggettive (autopuntura, igiene)

- Educare la persona assistita al controllo di peso corporeo, idratazione e dieta

- Coinvolgere il paziente nella valutazione periodica degli esami ematochimici

- Verificare i livelli di comprensione e l'efficacia dell'intervento educativo

- Intervenire tempestivamente $e$ in autonomia, pianificando interventi educativi mirati in relazione a specifici bisogni (dieta, farmaci...)

- Collaborare alle procedure relative alla messa in lista TX

- Controllare i referti ematochimici e strumentali e allertare il medico in caso di importanti alterazioni

Capacità di gestire le situazioni d'urgenza richieste dall'U.O. identificandone prontamente la tipologia e attivando i primi interventi appropriati

- Applicare prontamente e correttamente il protocollo per la gestione dell 'ipotensione intradialitica

- Identificare immediatamente situazioni di grave emergenza attivando correttamente le chiamate ai collaboratori in sala dialisi e agli specialisti
- Conoscere e applicare le procedure B.L.S. e B.L.S.D

- Utilizzare correttamente il carrello delle urgenze e $i$ relativi presidi

- Conoscere l'istruzione operativa relativa alla procedura di stacco del paziente dal circuito ematico in caso di eventi avversi

\section{Area critica}

Conoscere le attrezzature e il loro funzionamento

- Monitor per $i$ vari trattamenti

Conoscere i dispositivi delle varie attrezzature

- Circuiti, concentrati, soluzioni e filtri

- Osmosi inversa, preparati in sacche

Conoscere le tecniche per i trattamenti extracorporei

- Dialisi intermittenti (HD, HF)

- Dialisi continue (SCUF, CVVH, CVVHD, CVVHDF, SLED)

- Plasmaferesi selettive o semiselettive (PAP, PEX, Prometheus, MARS, CPFA)

- Emoperfusione

Capacità di organizzare l'esecuzione dei trattamenti dialitici a pazienti critici in contesti clinici diversi

- Gestire la presenza del personale nelle varie sedi in base ai trattamenti e alle competenze

- Assicurare la presenza del materiale nelle sedi dove si effettuano $i$ trattamenti

- Controllare le attrezzature(funzionamento-disinfezione) e la giacenza del materiale dialitico presente nelle varie sedi (quantitàscadenza-tipo)

- Eseguire i controlli degli impianti di trattamento delle acque

- Essere in grado di richiedere tempestivamente l'intervento dei tecnici in caso di malfunzionamento

- Gestire l'emergenza clinica, tecnica e organizzativ

Conoscere le patologie e le cause che richiedono il trattamento dialitico in area critica

- Patologie cardio-circolatorie

- Fase pre elo post trapianti

- Edema polmonare acuto

- Sepsi

- Patologie post trauma

- Patologie da intossicazioni

- Cause chirurgiche

- Insufficienza multi organo (mof)

- Patologie oncologiche

Conoscere le modalità di anticoagulazione

- Anticoagulazione sistemica con eparina continua elo intermittente

- Anticoagulazione locale con citrato

Gestire il trattamento dialitico nel paziente critico adulto e pediatrico nei vari contesti clinici

- Gestire la seduta dialitica secondo le indicazioni terapeutiche

- Accertare le condizioni cliniche e pianificare il trattamento 
- Monitorare i parametri vitali dell'assistito, utilizzare i sistemi di controllo delle apparecchiature e segnalare le variazioni meritevoli di altri interventi

- Sorvegliare visivamente la persona assistita per riconoscere preventivamente segni e sintomi di eventi

- Sorvegliare visivamente il circuito per riconoscere tempestivamente eventuali anomalie

- Gestire in modo accurato gli accessi vascolari disponibili

- Prevenire e gestire le eventuali complicanze secondo i protocolli definiti

- Comunicare con il paziente in relazione alle condizioni clinico-cognitive di quest'ultimo

\section{Capacità di registrare e di gestire $\mathbf{i}$ dati}

- Registrare l'andamento della seduta (parametri, eventi, interventi e valutazione)

- Utilizzare un sistema di reporting anche informatizzato

- Utilizzare sistemi di monitoraggio in remoto delle postazioni

- Utilizzare sistemi per la raccolta dei dati di attività

Capacità di lavorare in team multi professionali e multi disciplinari

- Collaborare quando possibile per la pianificazione e la programmazione dei trattamenti sulla base delle esigenze cliniche e organizzative

- Realizzare e applicare strumenti operativi (istruzioni, procedure e protocolli) condivisi

- Diffondere le conoscenze relative alle Linee Guida internazionali e alle evidenze disponibili

- Partecipare a incontri di team

- Realizzare eventi formativi per l'acquisizione e l'aggiornamento delle competenze per i professionisti dei vari contesti clinici

\section{Discussione}

Le competenze citate nelle singole aree possono/devono essere riallocate in base alle singole realtà organizzative e operative, potendo, però, essere utilizzate come linea di indirizzo per i centri che intendono implementare o riorganizzare i servizi offerti.

In ciascuna delle aree citate sono prevedibili, e, a volte, già effettivi, ruoli di avanzata competenza: figure come il "Professional", presente in Toscana, o la figura del "Case Manager", presente in Emilia Romagna, l'Infermiere dedicato nella DP, il Referente degli accessi vascolari, il Referente delle piaghe difficili e il Coordinatore di trapianto presente in alcune strutture IRCSS. Per non parlare di altre potenziali aree già occupate dall'infermieristica oltralpe, come l'Infermiere referente per l'anemia e il Pratictioner, con possibilità di prescrizione di farmaci già in trattamento. Nel presente documento non é stata trattata l'area della nefrologia pediatrica, che sarà sviluppata a breve in collaborazione alcuni colleghi del settore, che rappresenta una specificità trasversale a tutte le aree qui individuate. Il campo operativo è ampio, variegato nelle potenzialità sia di collaborazione strutturata e intelligente che di autonomie organizzative e relazionali.

\section{Conclusioni}

Questo elaborato vuole essere un iniziale contributo a un percorso di competenze infermieristiche nell'area nefrologica, nella consapevolezza che l'azione infermieristica è discriminante rispetto all'efficienza e all'efficacia dei percorsi diagnostici, terapeutici e assistenziali in tutte le aree citate.

Essere professionisti comporta un salto di qualità nella gestione sia delle conoscenze che delle relazioni.

I benefici di una professione infermieristica liberata dal1"“ausiliarità" medica sono molti e tangibili sia in termini economici, per il Servizio Sanitario Nazionale, che per il cittadino, in termini di umanizzazione/personalizzazione del servizio, della relazione di cura e dell'educazione alla salute.

\section{Riassunto}

L'articolo esprime la posizione attuale dell'associazione EDTNA/ERCA Filiale Italiana rispetto al dibattito sulle competenze infermieristiche avanzate - o specialistiche in atto nella comunità medico-sanitaria nazionale. Facendo riferimento al modello ISFOL, come strumento della definizione delle competenze nel mondo del lavoro, nel territorio europeo ed oltre, il modello viene declinato per il mondo sanitario ed ulteriomente capillarizzato per le aree di assistenza infermieristica nefrologica. Il modello riconosce competenze di base, competenze di settore, trasversali e specifiche. Attraverso questo percorso, da generico e a specifico, vengono riconosciute 6 aree specifiche di assistenza nefrologica (ambulatorio, degenza, dialisi peritoneale, emodialisi, area critica, trapianti) ed in ognuna di esse vengono elencate le competenze di pertinenza infermieristica (sapere e saper fare, saper essere). L'elaborato si propone come strumento di riferimento, di confronto e di stimolo per le varie realtà operative nazionale dell'assistenza nefrologica.

Parole chiave: Compentenze infermieristiche, Assistenza nefrologica

Dichiarazione di conflitto di interessi: Gli Autori dichiarano di non avere conflitto di interesse.

Contributi economici agli Autori: Gli Autori dichiarano di non aver ricevuto sponsorizzazioni economiche per la preparazione dell'articolo.

Indirizzo degli Autori:

Dr.ssa Marisa Pegoraro

EDTNA/ERCA Filiale Italiana

c/o DIALISI CAL Corsico

Via Travaglia 5

20094 Corsico (MI)

marisa_pegoraro@fastwebnet.it 


\section{Bibliografia}

1. Massai D, Amerini A, Borgellini S, Brugnoli S. L'infermiere 4/2007. Inserto - Speciale competenze: la valutazione. s.1.: IPASVI, l'infermiere 4/2007.

2. Kuntzle W. EDTNA/ERCA 1992. Core Curriculum for post basic education. EDTNA/ERCA 1992.

3. Benner P, ed. italiana a cura di C. Calamandrei, L. Rasero, 2003. L'eccellenza nella pratica clinica dell'infermiere. s.l.: McGrawHill, ed. italiana a cura di C. Calamandrei, L. Rasero, 2003.

\section{Bibliografia di approfondimento}

1. Bottio C, Guerrieri C. Il tutor clinico. Manuali Franco Angeli Ed. 2011.

2. Centro di eccellenza per la cultura e la ricerca infermieristica. Costruzione di un modello per la valutazione delle competenze infermieristiche nell'esame di abilitazione professionale. IPASVI, Roma 2013.
3. CNT. Documento informativo sul programma di trapianto di rene da donatore vivente - Centro Nazionale Trapianti 2009.

4. Mahon A, Jenkins K. Insufficienza Renale Cronica (stadio 1-3), una guida per la pratica clinica, EDTNA/ERCA 2007, Ed. Italiana 2008.

5. Mahon A, Jenkins K. Insufficienza Renale Cronica (stadio 4-5), una guida per la pratica clinica, EDTNA/ERCA 2008, Ed. Italiana 2010.

6. Trevitt R. Renal Transplantation, A guide to Clinical Practice. EDTNA/ERCA 2009. Ed. italiana disponibile in formato elettronico sul sito www.edtna-erca.it.

7. Riemann A, Casal MC. Peritoneal Dialysis, A guide to Clinical Practice, EDTNA/ERCA 2009, Ed. italiana disponibile in formato elettronico sul sito www.edtna-erca.it.

8. Jenkins K, Bennett L. Conservative Management in Advanced Kidney Disease, A guide to Clinical Practice. EDTNA/ERCA 2011.

9. Albarran JW, Saraiva M. Acute Kidney Ingiury, A guide to Clinical Practice. EDTNA/ERCA 2012.

10. Kalliopi AP, Nutritional Care for Adults with Chronic Kidney Disease, A guide to Clinical Practice. EDTNA/ERCA 2012.

11. Hoo TM, Marin M. Drugs in Renal Replacement therapy, A guide to Clinical Practice. EDTNA/ERCA 2012. 